\title{
Convex and Radially Concave Contoured Distributions
}

\author{
Wolf-Dieter Richter \\ Institute of Mathematics, University of Rostock, Ulmenstraße 69, Haus 3, 18057 Rostock, Germany \\ Correspondence should be addressed to Wolf-Dieter Richter; wolf-dieter.richter@uni-rostock.de
}

Received 24 June 2015; Revised 18 October 2015; Accepted 21 October 2015

Academic Editor: Z. D. Bai

Copyright (C) 2015 Wolf-Dieter Richter. This is an open access article distributed under the Creative Commons Attribution License, which permits unrestricted use, distribution, and reproduction in any medium, provided the original work is properly cited.

\begin{abstract}
Integral representations of the locally defined star-generalized surface content measures on star spheres are derived for boundary spheres of balls being convex or radially concave with respect to a fan in $\mathbb{R}^{n}$. As a result, the general geometric measure representation of star-shaped probability distributions and the general stochastic representation of the corresponding random vectors allow additional specific interpretations in the two mentioned cases. Applications to estimating and testing hypotheses on scaling parameters are presented, and two-dimensional sample clouds are simulated.
\end{abstract}

\section{Introduction}

The families of multivariate Gaussian and elliptically contoured distributions have served for a long time as the main basis of numerous probabilistic models and their many successful applications. Basics of estimation theory of elliptically contoured distributions can be found in [1-3] and, for example, [4]. Advancing needs of statistical practice as well as longstanding challenging mathematical questions stimulated the development of larger classes of probability laws containing many well known distributions as particular elements. We note that [5] surveys a big part of the distribution theory in $\mathbb{R}^{2}$. Numerous authors contributed to establishing the class of multivariate star-shaped distributions. For a recent review of this development, see [6]. Estimating level sets of star-shaped densities has been dealt with in [7-10].

Several aspects of analyzing a cloud of sample points may be of importance for the process of defining a class of probability laws. The visual impression of the appearance of star-shaped figures built by the points of a sample cloud may lead to the idea that the boundaries of star-bodies, henceforth called star-spheres, represent density level sets of a probability law. Counting the sample points belonging to thin layers about star-spheres then leads to the idea that a certain function that assigns a nonnegative number to every such star-sphere serves as the density generating function (dgf) of a nonnegative random variable (rv) or, more generally, as a function being proportional to the Radon-Nikodym density of a multivariate probability law with respect to a certain $\sigma$-finite measure defined on the sample space. We call such a function a univariate level density function of the multivariate probability distribution.

The combination of the aspects of defining level sets of a multivariate density and of assigning a nonnegative level to every such set will be reflected here in a new method of integration. This method may be considered as a heightening and generalization of the classical principle of Cavalieri which was modified by Torricelli. Combining integration on level sets with that along the levels may also be considered as a geometric disintegration method. This method is essentially based upon certain non-Euclidean surface measures on starspheres. It is one of the main aims of this paper to further develop this theory for two important types of star-spheres. Convex bodies and star-bodies being radially concave with respect to a fan in $\mathbb{R}^{n}$ build these two classes of star-bodies. Thus, in this paper, the focus is on considering probability laws having the boundary of such sets as their density level sets or their contour sets. Actually, the results in Section 3 are mainly restricted to, possibly shifted, symmetric contour sets being norm or antinorm spheres.

There are different ways to introduce a dfg of a continuous probability law. Looking through the statistical and mathematical literature, one finds many interesting nonnegative and suitably integrable functions which may serve as a dfg. Another way to introduce a dfg is to analyze the structure of a known multivariate density and to extract from it, if possible, 
a function which does not depend on the surface measure on the star-spheres but depends exclusively on the levels of the multivariate density.

It is well known that the definition of a dfg is not unique and so is how to deal with this circumstance. Densities with heavy tails may be of interest in (re)insurance, and densities with light tails may be of interest in reliability theory. Both types of densities can be modeled using each time a suitable dfg.

Modeling the density level sets and the univariate level density of a multivariate distribution can be done in a combined way or separately from each other. Sometimes a parameter may influence both the level density and the density contour sets of a multivariate distribution. Another parameter may be only for one of these two aspects of importance.

The paper is organized as follows. Some basic facts from the theory of star-shaped distributions, with an emphasis on geometric measure representations, are collected in Section 2. New geometric descriptions of surface measures on boundary spheres of balls being radially concave with respect to a fan in $\mathbb{R}^{n}$, or convex, are presented in Section 3. Moreover, new statistical applications of geometric measure representations of norm and antinorm contoured distributions and of stochastic representations of correspondingly distributed random vectors are discussed there. In particular, distributions are illustrated by simulated sample clouds. The basics for estimating and testing hypothesis on scaling parameters are presented at the end of Section 3. Section 4 deals with proving the new results, and a discussion of the results can be found in the final Section 5 .

\section{Star-Shaped Distributions}

Geometric measure representations and stochastic representations of corresponding random vectors have been proved in [6] for general star-shaped distributions making essentially use of the notion of a star-generalized surface content measure. The latter is defined in a local way by taking derivatives of sector volumes and is known to be equivalently defined in an integral (in dimension two even explicitly differential geometric) way in the cases of $l_{n, p}$-spheres and ellipsoids. For recent results and a survey of their probabilistic and statistical applications we refer to [6]. Here, some basic facts from star-shaped distribution theory and its applications will be summarized.

Let a random vector $Y$ follow the probability density function (pdf):

$$
\varphi_{g, K, \nu}(x)=C(g, K) g\left(d_{K}(x-v)\right), \quad x \in \mathbb{R}^{n},
$$

where $v \in \mathbb{R}^{n}$ is a vector of location, $K \subset \mathbb{R}^{n}$ is a starbody having the origin as an inner point, $d_{K}$ is the distance function, or Minkowski functional, of the star-body $K$,

$$
d_{K}(x)=\inf \{\lambda \geq 0: x \in \lambda K\}, \quad x \in \mathbb{R}^{n},
$$

the function $g:[0, \infty) \rightarrow[0, \infty)$ satisfies $0<I(g)<\infty$, where $I(g)=\int_{0}^{\infty} r^{n-1} g(r) d r$, and the normalizing constant allows the representation

$$
C(g, K)=\frac{1}{\mathfrak{D}_{S}(S) I(g)} .
$$

Assuming that the technical Assumption 1 in [6] is satisfied which deals with a certain smoothness property of the boundary $S$ of $K, \mathfrak{D}_{S}$ denotes the star-generalized surface content measure defined on the Borel subsets of $S$. The probability measure corresponding to $\varphi_{g, K, \nu}$ allows the geometric measure representation or disintegration formula:

$$
\begin{array}{r}
\Phi_{g, K, v}(B) \\
=C(g, K) \int_{0}^{\infty} r^{n-1} g(r) \mathfrak{D}_{S}\left(\left[\frac{1}{r}(B-v)\right] \cap S\right) d r, \\
B \in \mathfrak{B}^{n} .
\end{array}
$$

$K$ is called the density contour defining star-body of this distribution and any $g$ under consideration is a density generating function $(\mathrm{dfg})$. The sets $(B-\nu) \cap S(r)$ with $S(r)=r S$ may be considered playing the role of the indivisibles within a generalized principle of Cavalieri (which was modified by Torricelli). The random vector $Y$ satisfies the stochastic representation:

$$
Y \stackrel{d}{=} R \cdot U_{S}
$$

where $R$ and $U_{S}$ are stochastically independent, $R$ has the pdf

$$
f_{R}(r)=I_{(0, \infty)}(r) \frac{1}{I(g)} r^{n-1} g(r),
$$

and $U_{S}$ follows the star-generalized uniform probability distribution $\omega_{S}$ on the Borel- $\sigma$-field $\mathscr{B}_{S}=\mathscr{B}^{n} \cap S$ over $S$, $U_{S} \sim \omega_{S}$, and

$$
P\left(U_{S} \in A\right)=\frac{\mathfrak{D}_{S}(A)}{\mathfrak{D}_{S}(S)}, \quad A \in \mathscr{B}_{S} .
$$

Because of (7), $U_{S}$ is called the star-generalized uniform basis of $Y$. The symbol $X \stackrel{d}{=} Y$ means that the random vectors $X$ and $Y$ follow the same probability law while $X \sim Q$ indicates that the random vector $X$ follows the probability distribution Q.

For $A \in \mathscr{B}_{S}$, we introduce the central projection cone,

$$
\operatorname{CPC}(A)=\left\{x \in \mathbb{R}^{n}: \frac{x}{h_{K}(x)} \in A\right\},
$$

and the star sector of star radius $r$,

$$
\operatorname{sector}(A, r)=\operatorname{CPC}(A) \cap K(r),
$$

where

$$
K(r)=r \cdot K=\left\{\left(r x_{1}, \ldots, r x_{n}\right)^{T}:\left(x_{1}, \ldots, x_{n}\right)^{T} \in K\right\}
$$


is a star ball of star radius $r$. Let $\mu$ be the Lebesgue measure in $\mathbb{R}^{n}$. Then the star-generalized surface measure is defined on $r \mathscr{B}_{S}$ in a local approach by

$$
\mathfrak{D}_{S}(A)=f^{\prime}(r) \quad \text { where } f(r)=\mu(\operatorname{sector}(A, r)) \text {. }
$$

Making use of the star-sphere intersection proportion function (ipf) of a set $A$,

$$
\mathfrak{\mho}_{S}(A, r)=\omega_{S}\left(\left[\frac{1}{r} A\right] \cap S\right), \quad r>0,
$$

the disintegration representation of $\Phi_{g, K, v}$ may be written as

$$
\Phi_{g, K, v}(B)=\frac{1}{I(g)} \int_{0}^{\infty} r^{n-1} g(r) \mathfrak{F}_{S}(B-\nu, r) d r,
$$

$$
B \in \mathscr{B}^{n} \text {. }
$$

The most immediate applications of this formula appear in cases where the ipf is a constant or an indicator of an interval. If, for a certain set $B$, the ipf takes a constant value, $C$, say, then $\Phi_{g, K, v}(B)$ is just equal to this value $C$.

If, for a statistic $T, B(t)=\{T<t\}, t \in \mathbb{R}$, and the ipf of all sets $B(t)$ take the constant value, $C(t)$, say, then the statistic $T$ is robust with respect to the $\mathrm{dfg} g$; that is, the distribution of $T$ does not depend on $g$.

If, for a certain set $A(x)$, the ipf is the indicator function of an interval, $[0, b(x)]$, say, then

$$
\frac{d}{d x} \Phi_{g, K, v}(A(x))=\frac{b^{\prime}(x) b(x)^{n-1} g(b(x))}{I(g)}
$$

For specific statistical examples of such type we refer to Section 3. Applications of (13) in cases where the ipf is more structured are often more involved. Such a situation will be considered in Example 7.

The main aim of this paper, however, is not only to give attractive examples where the geometric measure representation applies but also to give nontrivial explanations of the locally defined surface measure $\mathfrak{D}_{S}$ on the basis of an integral (or differential geometric) approach. This will be done in the first two parts of Section 3 for the two important cases where $K$ is a norm or antinorm ball. As a result, in formulas (4), (5), (7) and (13), $\mathfrak{D}_{S}$ will afterwards allow additional specific integral (or differential geometric) interpretations in the two mentioned cases. The class

$$
\begin{aligned}
& \text { CStSh }=\left\{\Phi_{g, K, \nu}: \nu \in \mathbb{R}^{n}, K \text { is a star body with } 0\right. \\
& \quad \in \operatorname{int} K, g \text { is a dgf }\},
\end{aligned}
$$

where int $K$ means the interior of $K$, is called the class of continuous star-shaped distributions. A random vector $Y$ is said in [6] to belong to the bigger class of star-shaped distributions $S t S h^{(n)}$ if there are a vector $v \in \mathbb{R}^{n}$, a star-body $K$ with $0 \in$ int $K$ (and boundary $S$ ), and a nonnegative random variable (rv) $R$ with cumulative distribution function (cdf) $F$ such that $Y-v \stackrel{d}{=} R \cdot U_{S}$ where $U_{S} \sim \omega_{S}$ and $R$ and $U_{S}$ are independent. In this case, we write

$$
\begin{aligned}
& Y \sim \Psi_{F, K, \nu}, \\
& S t S h^{(n)}=\left\{\Psi_{F, K, \nu}: F\right. \text { is the cdf of a non-negative rv, }
\end{aligned}
$$

$$
\left.K \text { is a star body, } 0 \in \operatorname{int} K, v \in \mathbb{R}^{n}\right\} \text {. }
$$

The random vector $U_{S}$ is called the star-generalized uniform basis of the class $S t S h^{(n)}$. If $K$ is symmetric with respect to the origin, $K=-K$, then the functional $d_{K}$ is a norm, $d_{K}=\|\cdot\|_{K}$, if $K$ is convex, and is an antinorm, $d_{K}=$ $+\cdot t_{K}$, if $K$ is radially concave with respect to a fan in $\mathbb{R}^{n}$. For the latter notions, see [11]. Note that $l_{n, p}$-symmetric distributions are norm or antinorm contoured if $p \geq 1$ or $0<p \leq 1$, respectively. We will study general convex or norm contoured distributions in Section 3.1 and distributions being radially concave with respect to a fan in $\mathbb{R}^{n}$, or antinorm contoured, in Section 3.2. The main aim of these two sections is to give closer descriptions of $\mathfrak{D}_{S}$ being basic for both the general stochastic representation of $Y$ in (5) and the specific geometric measure representations of $\omega_{S}$ in $(7)$ and $\Phi_{g, K, v}$ in (4) and (13) in case $Y$ has a density. Moreover, twodimensional distributions are illustrated by graphics showing simulated sample clouds. Applications to estimating and testing hypotheses on scaling parameters are demonstrated in Section 3.3. The proofs of the results from Sections 3.1 and 3.2 will be presented in Section 4, and a final discussion of the results follows in Section 5.

\section{Results}

We start the presentation of new results with a remark on asymmetric distribution laws which seems to be very useful: a distribution being star-shaped with respect to a fan $\mathfrak{F}$ may be restricted to arbitrary unions of elements of $\mathfrak{F}$.

Remark 1. Let $C_{i} \in \mathfrak{F}, i \in I$ and $C=\bigcup_{i \in I} C_{i}$. Then

$$
\begin{aligned}
& \Phi_{g, K, \nu}^{C}(B) \\
& \quad=\frac{\mathfrak{D}_{S}(S)}{\mathfrak{D}_{S}(S \cap C)} \frac{1}{I(g)} \int_{0}^{\infty} r^{n-1} g(r) \mathfrak{\mho}_{S}(B-\nu, r) d r
\end{aligned}
$$

is a probability law on $C \cap \mathfrak{B}^{n}$.

Here, $I$ is a suitable index set. The proof of this result follows immediately by conditioning.

We call the collection of all such distributions the class of fan restricted star laws and denote it by StL:

$$
S t L=\left\{\Phi_{g, K, v}^{C}, C=\bigcup_{i \in I} C_{i}, C_{i} \in \mathfrak{F}, \forall i\right\} .
$$

Elements of this distribution class are not symmetric, in general.

3.1. Norm Contoured Distributions. Let $K$ be convex and symmetric with respect to the origin throughout this section. 


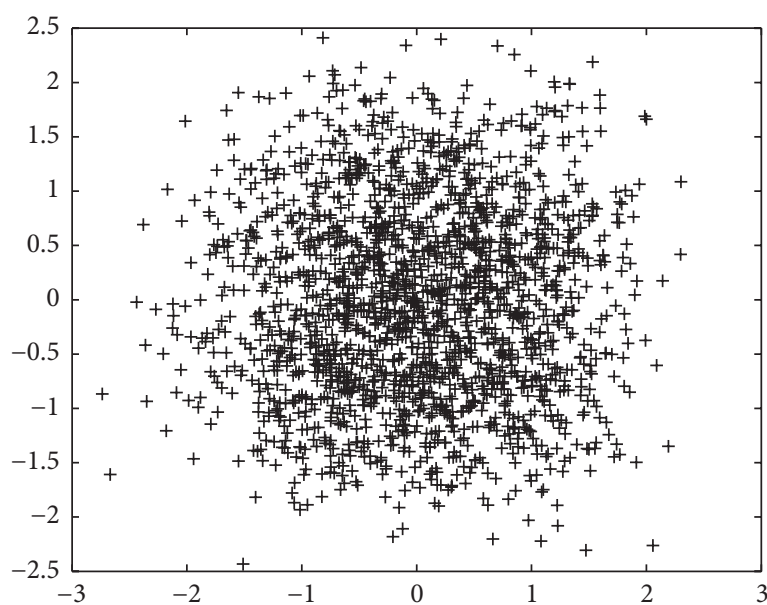

(a) $p=3$

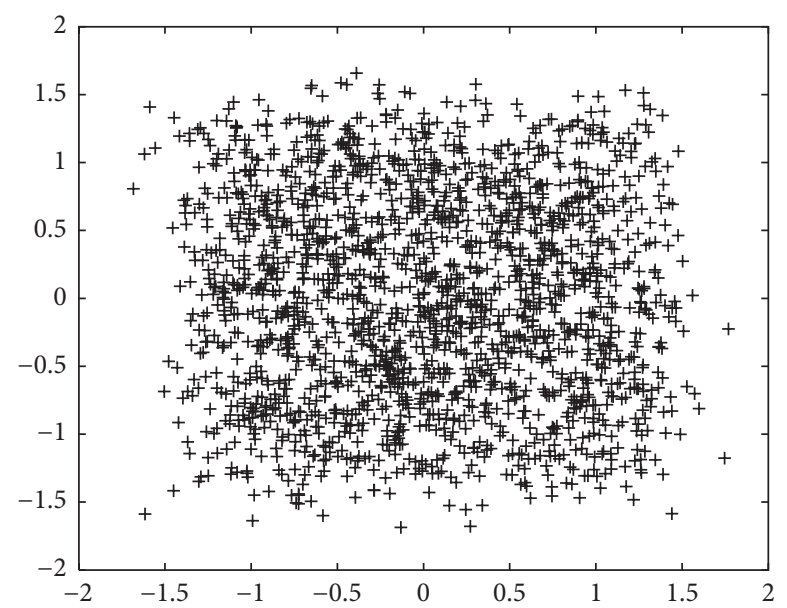

(b) $p=6$

FIGURE 1: Sample clouds of convex contoured $p$-generalized normal distributions $(n=2)$.

Our consideration is restricted therefore here to the class of norm contoured distributions:

$$
\begin{aligned}
& \mathcal{N} \mathscr{C}=\left\{\Psi_{F, K, v}\right. \\
&\left.\in S t S h^{(n)}: K \text { is the unit ball of a norm in } \mathbb{R}^{n}\right\} .
\end{aligned}
$$

Let the system of Borel sets from the upper half of the sphere $S$ be $\mathscr{B}_{S}^{+}$. For $A \in \mathscr{B}_{S}^{+}$, put

$$
\begin{aligned}
& G(A) \\
& \quad=\left\{\vartheta \in \mathbb{R}^{n-1}: \exists \eta=\eta(\vartheta) \text { with }\left(\vartheta^{T}, \eta\right)^{T} \in A\right\},
\end{aligned}
$$

and denote, wherever it exists, the outer normal vector to the norm sphere $S$ at the point $\left(\vartheta^{T}, \eta\right)^{T} \in S$ by $N(\vartheta)$. Wherever the outer normal vector is not defined, let $N(\vartheta)$ denote the zero element of $\mathbb{R}^{n}$. Note that the set of boundary points of $K$ where $\nabla \eta$ does not exist is countable and thus without any influence on the value of the integral in the following theorem. We recall that the surface content measure $\mathfrak{D}_{S}$ was locally defined in (11).

Theorem 2. In formulas (4), (5), (7) and (13), the surface content measure $\mathfrak{D}_{S}$ allows the representation

$$
\mathfrak{D}_{S}(A)=\int_{G(A)} d_{K^{*}}(N(\vartheta)) d \vartheta, \quad A \in \mathscr{B}_{S}^{+},
$$

where $K^{*}$ is the unit ball of the norm $\|\cdot\|^{*}$ being dual to $d_{K}=$ $\|\cdot\|_{K} \cdot$

We will refer to this result as to the integral or differential geometric approach to measuring surface content on a norm sphere based upon the dual norm geometry. We mention that a similar representation of $\mathfrak{D}_{S}(A)$ follows for arbitrary $A \in \mathscr{B}_{S}$. Due to Theorem 2 , if $K$ is convex, the surface measure $\mathfrak{D}_{S}$ henceforth allows both the local and the integral interpretation in formulas (4), (5), (7) and (13). Moreover,
Theorem 2 reflects a certain specific aspect of duality theory for norms.

In the next section we will deal in an analogous way with balls being radially concave with respect to a fan in $\mathbb{R}^{n}$.

Figures 1-3 show sample clouds of size $k=2000$ of $p$ generalized Gaussian distributed two-dimensional $(n=2)$ random vectors for different choices of $p, p \geq 1$. Notice that the six frames reflect different scaling of the clouds due to different values of $p$. While the sample cloud in Figure 1(a) might seem to be similar to the illustration of the Gaussian case the shape of the sample cloud approaches that of an axesaligned square if $p$ increases (or even tends to infinity). At the same time, the cloud (probability mass) becomes more and more concentrated. If, however, $p \geq 1$ is tending to one then the shape of the sample cloud approaches that of the diamond. At the same time, probability mass becomes much less concentrated and the contour of the sample cloud appears to be not as sharp as in the opposite case. Hence, the parameter $p$ of such a distribution might be called a shapeconcentration parameter. Note that Figures 6-8 also present sample clouds of convex contoured distributions but where emphasis is, inter alia, on the effect forced by an increasing sample size $k$.

Finally, let us remark that Figure 3(b) with equal rights could be presented in the next section because $l_{n, p}$-balls are both convex and radially concave with respect to the standard fan in $\mathbb{R}^{n}$ if $p=1$.

3.2. Antinorm Contoured Distributions. Throughout the present section, let $K$ denote a star-body having a positive and continuous radial function and being symmetric with respect to the origin and radially concave with respect to a fan $\mathfrak{F}=\left\{C_{1}, C_{2}, \ldots\right\}$ in $\mathbb{R}^{n}$. The Minkowski functional or distance function of $K$ is then an antinorm, $d_{K}=+\cdot+$, that is, a continuous, positively homogeneous, nondegenerate, and in $\mathfrak{F}$ super additive function. For more details we refer 


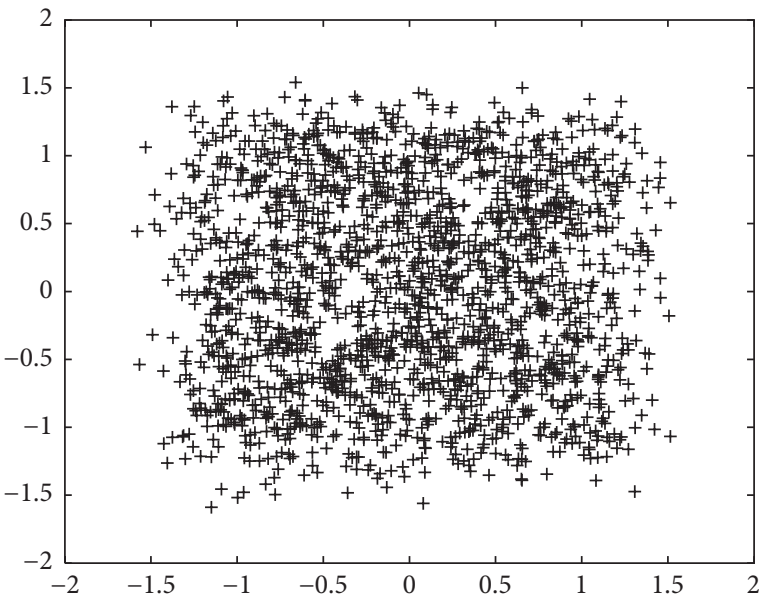

(a) $p=8$

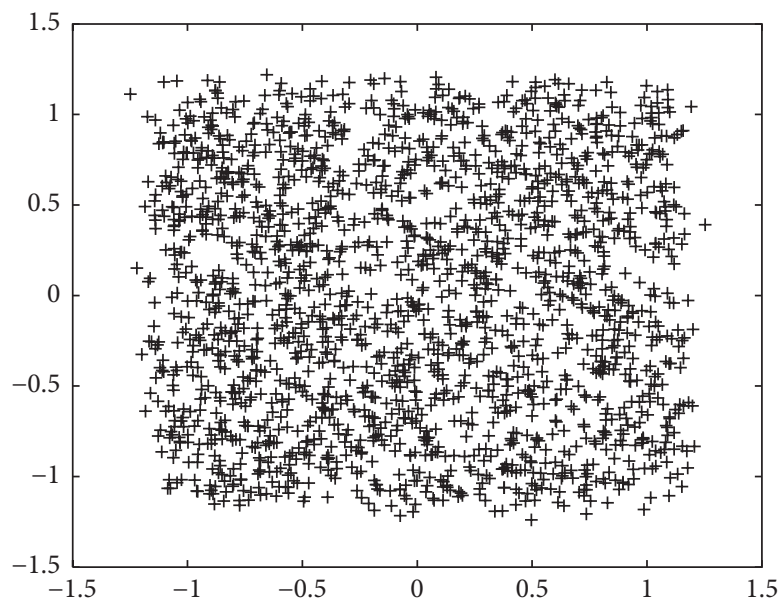

(b) $p=20$

Figure 2: Convex contoured cases far from the normal one.

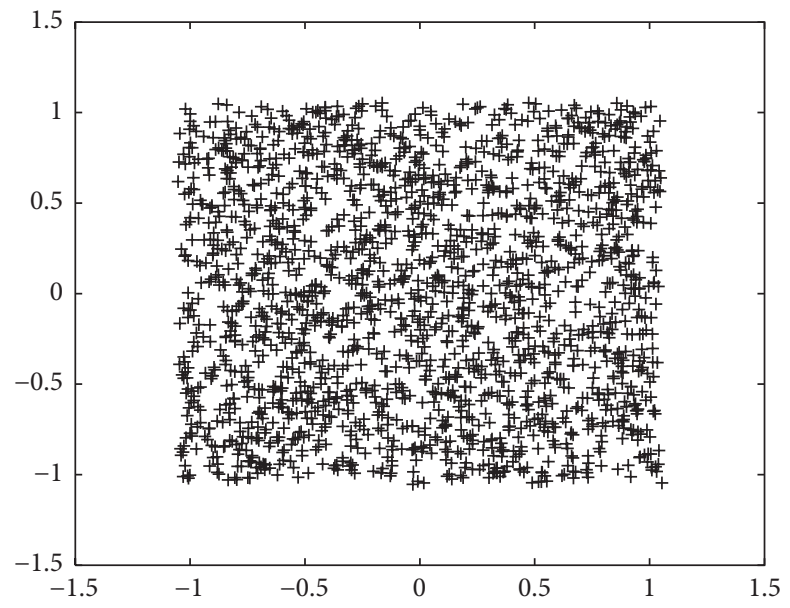

(a) $p=100$

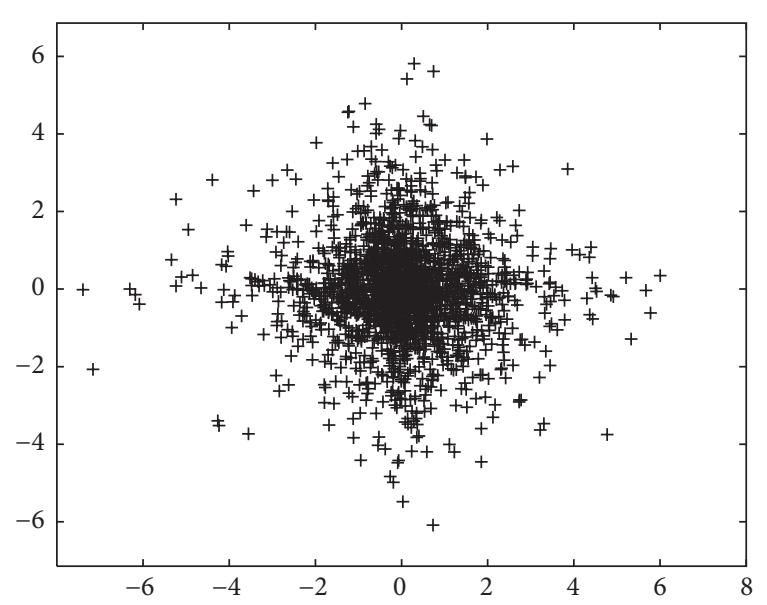

(b) $p=1$

Figure 3: Two extremal convex cases.

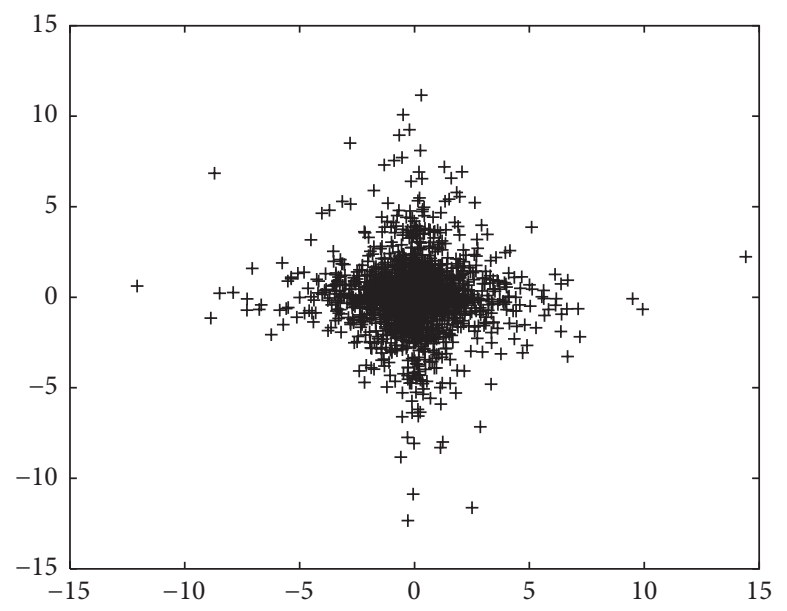

(a) $p=3 / 4$

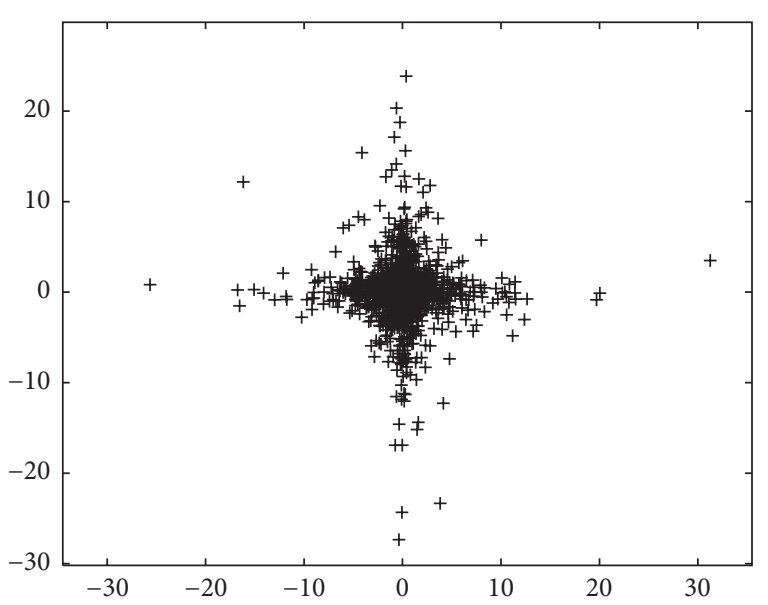

(b) $p=1 / 2$

FIGURE 4: Sample clouds of radially concave contoured $p$-generalized normal distributions. 


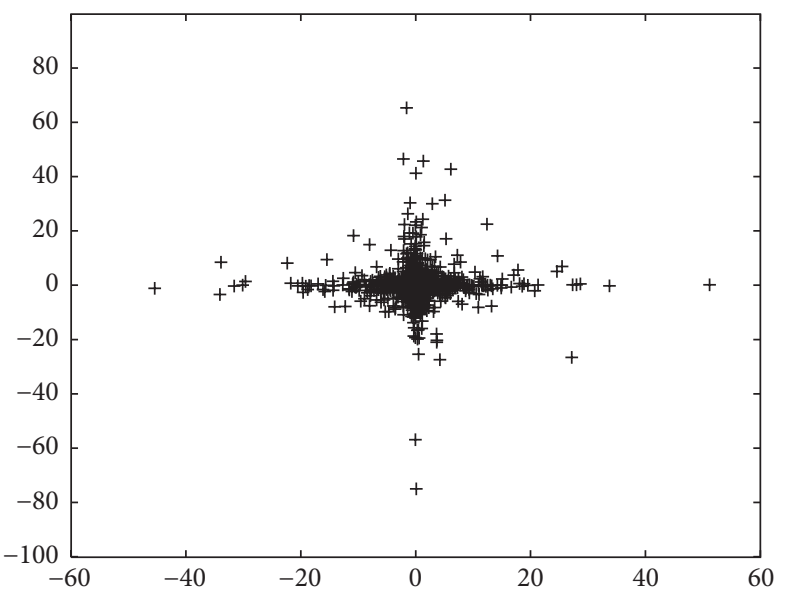

(a) $p=1 / 3$

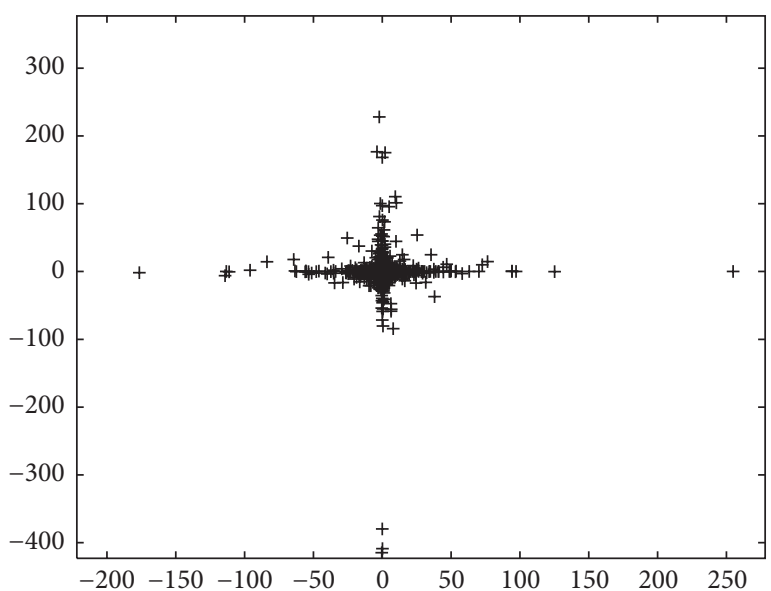

(b) $p=1 / 5$

FIGURE 5: Far reaching tails.

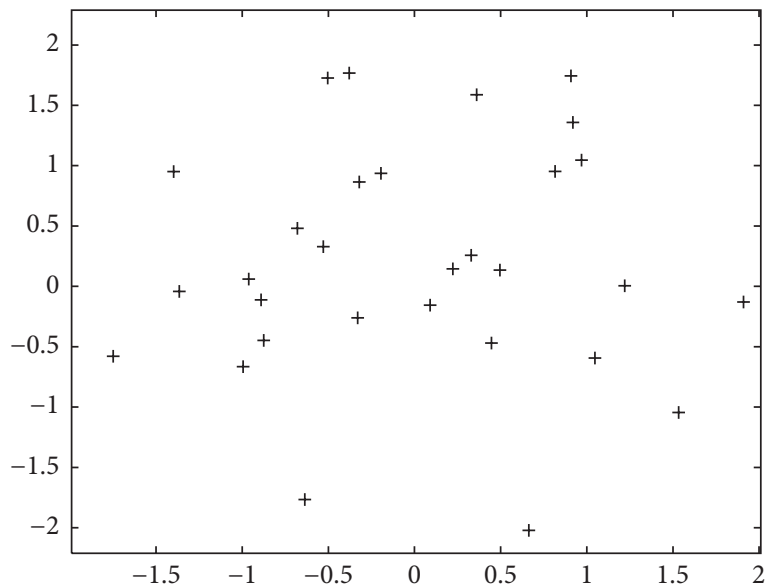

(a) $p=2$

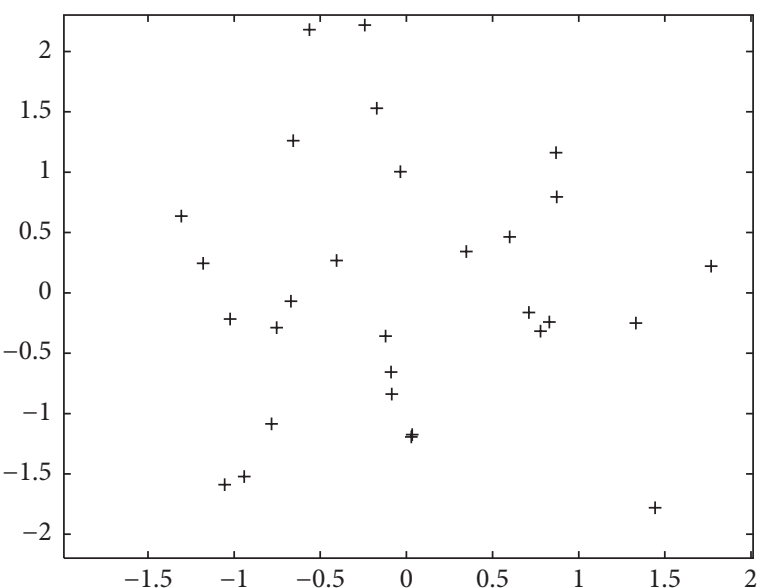

(b) $p=2,388677$

FIGURE 6: Generalized normal distribution: (small) sample size $k=30$.

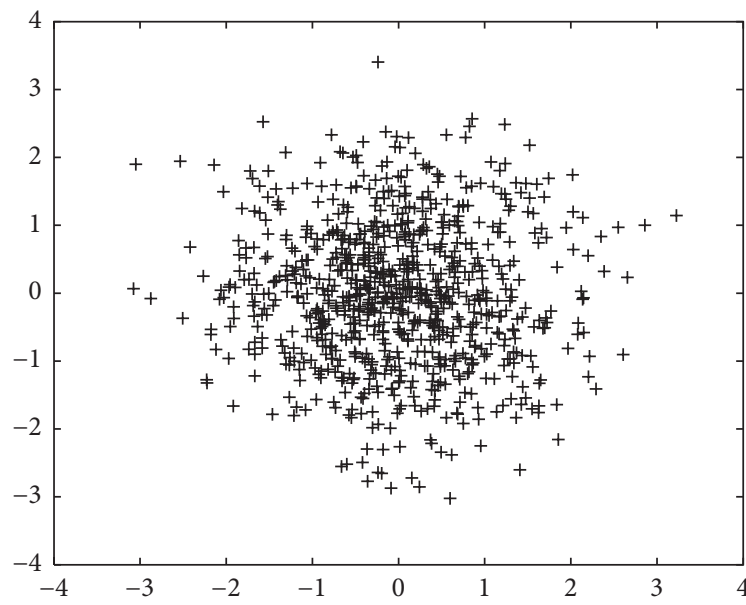

(a) $p=2$

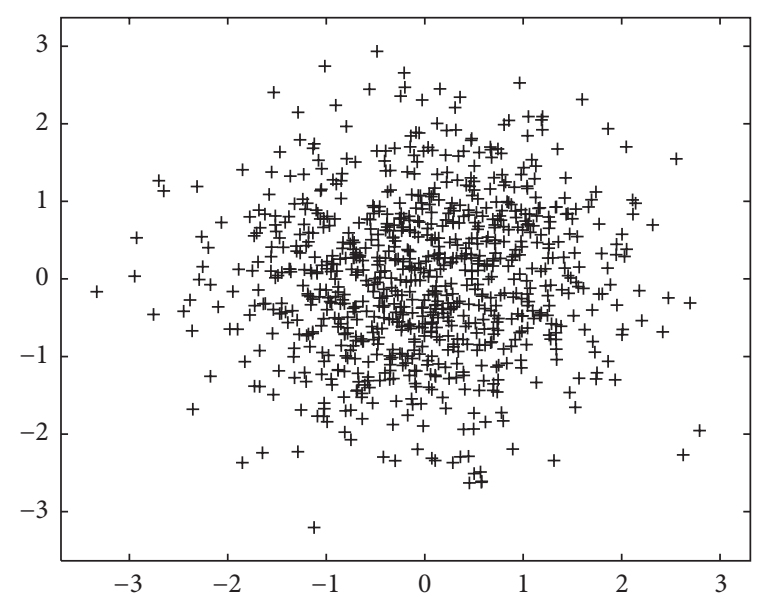

(b) $p=2,388677$

FIGURE 7: Sample (medium) size $k=850$. 


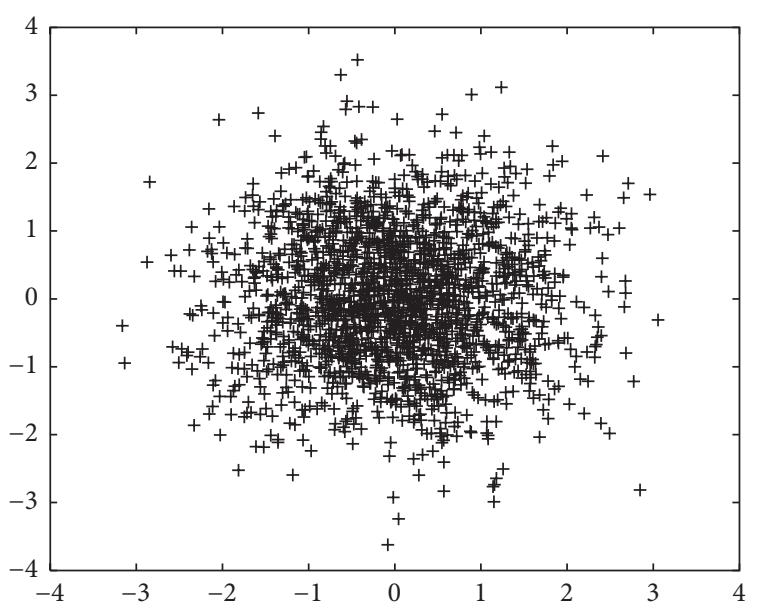

(a) $p=2$

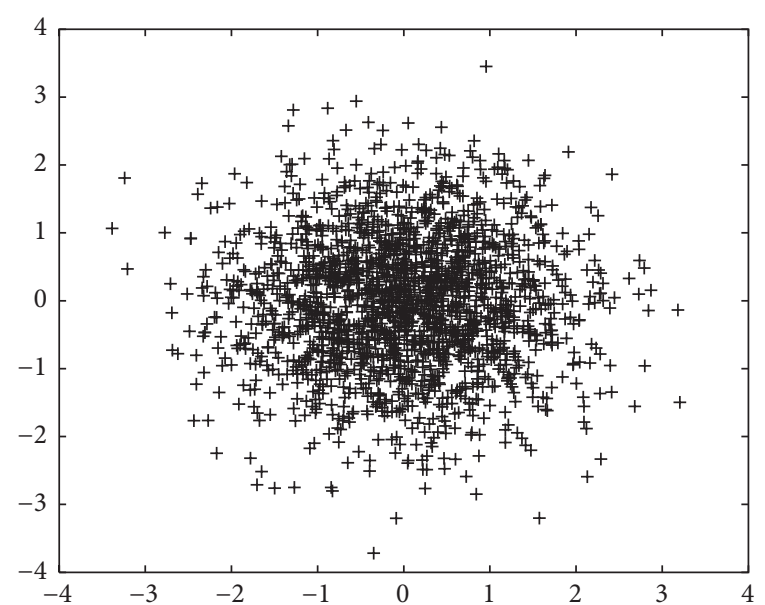

(b) $p=2,388677$

Figure 8: Sample (large) size $k=2000$.

to [11]. According to all the assumptions made so far, our consideration is restricted here to the class of antinorm contoured distributions:

$$
\mathscr{A} \cdot \mathcal{N} \mathscr{C}=\left\{\Psi_{F, K, v} \in S t S h^{(n)}:\right.
$$

$K$ is the unit ball of an antinorm in $\left.\mathbb{R}^{n}\right\}$.

Moreover, we assume that $K$ belongs to a particular class of antinorm balls, AN1, meaning that

(1) for every $i$ there is a 1-1-map $x_{S, i}: S^{(n-1)} \cap C_{i} \rightarrow$ $S \cap C_{i}$, where $S^{(n-1)}$ denotes the Euclidean unit sphere in $\mathbb{R}^{n}$,

(2) for every $i$, for all $u \in S^{(n-1)} \cap C_{i}$ there is a hyperplane $T(u)$ satisfying $T(u) \perp u$ and being an inner tangent plane to (the boundary of) $K$ at $x_{S, i}(u)$.

We define the antisupport function of $K$ with respect to $\mathfrak{F}$ by

$$
h_{K}^{\mathfrak{F}}(u)=\sum_{i} I_{C_{i}}(u) \inf \left\{u^{T} y: y \in S \cap C_{i}\right\}, \quad u \in \mathbb{R}^{n},
$$

and the antipolar set of $K$,

$$
K^{o}=\left\{\lambda(u) u: 0 \leq \lambda(u) h_{K}^{\mathfrak{F}}(u) \leq 1, u \in S_{E}^{(n-1)}\right\},
$$

where $0 \leq \lambda(u) \leq \infty$ if $h_{K}^{\mathfrak{\mho}}(u)=0$. Let $N(\vartheta)$ be the inner normal vector to $S$ at $\left(\vartheta^{T}, \eta\right)^{T} \in S$. In the sense of Remark 1 in [6], in what follows we will simply write

$$
\int_{G(A)} d_{K^{o}}(N(\vartheta)) d \vartheta \text { instead of } \sum_{i} \int_{G\left(A \cap C_{i}\right)} d_{K^{o}}(N(\vartheta)) d \vartheta .
$$

Theorem 3. In formulas (4), (5), (7) and (13), the surface content measure $\mathfrak{D}_{S}$ allows the representation

$$
\mathfrak{D}_{S}(A)=\int_{G(A)} d_{K^{o}}(N(\vartheta)) d \vartheta, \quad A \in \mathscr{B}_{S}
$$

In addition to the general local definition in formula (11), the result of this theorem allows the integral or differential geometric interpretation of the surface measure $\mathfrak{D}_{S}$ in the geometry having $K^{o}$ as its unit ball. Note that the special case where surface content of subsets of the boundary of the antinorm ball $K=B_{a, p}=\left\{x \in \mathbb{R}^{n}:\left(\sum_{i=1}^{n}\left|x_{i} / a_{i}\right|^{p}\right)^{1 / p} \leq\right.$ $1\}, 0<p \leq 1, a_{i}>0, \forall i$, is measured based upon the corresponding semiantinorm geometry has been dealt with already in [6].

Figures 5 and 6 show sample clouds of the same sample size and from the same distribution class as in Section 3.1 but with parameter $p$ chosen from the interval $(0,1)$. A big proportion of probability mass can be observed tending to the far tails of such distribution if $p$ is approaching zero. At the same time one can identify several points which could be considered being outliers if they had appeared under other circumstances. Hence, the parameter $p$ of such distribution might be called a shape-tail parameter.

3.3. Statistical Applications. This section deals with several examples where formula (13) applies. The first three examples present relatively immediate applications while the last example concerns a more advanced situation for calculating the ipf.

Example 4. Let $X_{1}, \ldots, X_{n}$ be independent $\mathrm{rv}$ following the common density of the power-exponential (or $p$-generalized Gaussian or $p$-generalized Laplace) distribution:

$$
\begin{aligned}
& f_{p}\left(x ; \mu, \sigma^{2}\right)=\frac{C_{p}}{\sigma} \exp \left\{-\frac{|x-\mu|^{p}}{p \sigma^{p}}\right\}, \\
& x \in \mathbb{R}, C_{p}=\frac{p^{1-1 / p}}{2 \Gamma(1 / p)},
\end{aligned}
$$

where the location parameter $\mu \in \mathbb{R}$ and the shape-concentration or shape-tail parameter $p>0$ are known and the 
scaling parameter $\sigma$ is unknown. The maximum-likelihood estimator (mle) of $\sigma$ is

$$
\widehat{\sigma}=\left(\frac{1}{n} \sum_{i=1}^{n}\left|X_{i}-\mu\right|^{p}\right)^{1 / p}
$$

and the random vector $X_{(n)}=\left(X_{1}, \ldots, X_{n}\right)^{T}$ follows the density $\varphi_{g_{P E}, K, v}$ where $v=(\mu, \ldots, \mu)^{T}, K=\sigma B_{p}, B_{p}=\left\{x \in \mathbb{R}^{n}\right.$ : $\left.|x|_{p} \leq 1\right\},|x|_{p}=\left(\sum_{i=1}^{n}\left|x_{i}\right|^{p}\right)^{1 / p}$, and

$$
g_{P E}(r)=\exp \left\{-\frac{r^{p}}{p}\right\}, \quad r \geq 0 .
$$

The set $B_{p}$ is convex if $p \geq 1$ and radially concave with respect to the standard fan in $\mathbb{R}^{n}$ if $0<p \leq 1$. Hence,

$$
\frac{1}{\sigma}\left(X_{(n)}-\nu\right) \sim \Phi_{g_{P E}, B_{p}, 0_{n}}
$$

follows a centered convex or radially concave contoured distribution if $p \geq 1$ or $0<p \leq 1$, respectively. It turns out that

$$
P\left(n\left(\frac{\widehat{\sigma}}{\sigma}\right)^{p}<t\right)=\Phi_{g_{P E}, B_{p}, 0_{n}}\left(t^{1 / p} B_{p}\right)
$$

and thus

$$
\begin{aligned}
& P\left(n\left(\frac{\widehat{\sigma}}{\sigma}\right)^{p}<t\right) \\
& \quad=\frac{1}{I\left(g_{P E}\right)} \int_{0}^{\infty} r^{n-1} g_{P E}(r) \mathfrak{F}_{S_{p}}\left(t^{1 / p} B_{p}, r\right) d r
\end{aligned}
$$

where $I\left(g_{P E}\right)=p^{n / p-1} \Gamma(n / p), \Gamma$ denotes the Gamma function, $S_{p}=\partial B_{p}$ is the $p$-sphere, that is, the set of boundary points of $B_{p}$, and the $p$-sphere ipf of $t^{1 / p} B_{p}$ is

$$
\mathfrak{F}_{S_{p}}\left(t^{1 / p} B_{p}, r\right)=I_{\left[0, t^{1 / p}\right]}(r), \quad r \geq 0 .
$$

Consequently,

$$
P\left(n\left(\frac{\widehat{\sigma}}{\sigma}\right)^{p}<t\right)=\frac{1}{I\left(g_{P E}\right)} \int_{0}^{t^{1 / p}} r^{n-1} g_{P E}(r) d r
$$

that is, $n(\widehat{\sigma} / \sigma)^{p}$ follows the $\chi_{g_{P E}}^{p}$-density with $n$ d.f. introduced in [12]:

$$
t \longmapsto \frac{t^{n / p-1} e^{-t / p}}{p^{n / p} \Gamma(n / p)}=f_{n, g_{P E}, p}^{\chi}(t), \quad t>0 .
$$

This exact distributional result allows constructing confidence intervals for and testing hypotheses on the scaling parameter $\sigma$.

Example 5. We consider independent $\mathrm{rv} X_{1}, \ldots, X_{n}$ following the densities

$$
f_{p}\left(x ; \mu_{i}, \sigma^{2} a_{i}^{2}\right)=\frac{C_{p}}{\sigma a_{i}} \exp \left\{-\frac{\left|x-\mu_{i}\right|^{p}}{p\left(\sigma a_{i}\right)^{p}}\right\}, \quad x \in \mathbb{R},
$$

where $\mu_{i} \in \mathbb{R}, p>0, a_{i}>0$ are known and $\sigma>0$ is an unknown common scaling parameter. The mle of $\sigma$ allows the functional representation

$$
\widehat{\sigma}=\frac{1}{n^{1 / p}}\left|X_{(n)}-v\right|_{a, p},
$$

where $X_{(n)}=\left(X_{1}, \ldots, X_{n}\right)^{T}, v=\left(\mu_{1}, \ldots, \mu_{n}\right)^{T}$, and

$$
|x|_{a, p}=\left(\sum_{i=1}^{n}\left|\frac{x_{i}}{a_{i}}\right|^{p}\right)^{1 / p}, \quad x \in \mathbb{R}^{n}
$$

The density of $X_{(n)}$ is

$$
f_{X_{(n)}}(x)=\frac{C_{p}^{n}}{\sigma^{n} a_{1} \cdots a_{n}} g_{P E}\left(d_{\sigma B_{a, p}}(x-\nu)\right),
$$

and thus

$$
P\left(n\left(\frac{\widehat{\sigma}}{\sigma}\right)^{p}<t\right)=\Phi_{g_{P E}, B_{a, p}, 0_{n}}\left(t^{1 / p} B_{a, p}\right),
$$

where $B_{a, p}=\left\{x \in \mathbb{R}^{n}:|x|_{a, p} \leq 1\right\}$. Note that $\mathfrak{F}_{S_{a, p}}\left(t^{1 / p} B_{a, p}, r\right)$ does not depend on $a$. Hence, $n(\widehat{\sigma} / \sigma)^{p}$ follows the $\chi_{g_{P E}}^{p}$ distribution with $n$ d.f., independently of $a=\left(a_{1}, \ldots, a_{n}\right)^{T}$.

While Examples 4 and 5 are restricted to the $p$ generalized Gaussian or Laplace distribution which is defined using the dfg $g_{P E}, p>0$, Example 6 deals with measuring the same sets as before but with measures having another level distribution, especially allowing lighter and heavier distribution tails. Such tails may be of interest in various types of applications.

Example 6. Let us assume that $Y$ follows the probability distribution law $\Phi_{g, K, v}$ with $v \in \mathbb{R}^{n}, K=B_{\sigma a, p}$ and dfg $g$. Examples of dfg are, besides $g_{P E}$, the Kotz type dgf $g_{K}$ defined by $g_{K}(r)=r^{M-1} e^{-\beta r^{\gamma}}, \beta, \gamma>0, M+n>1$, and the PearsonVII-type dfg $g_{P T 7}$ defined by $g_{P T 7}(r)=(1+r / m)^{-M}, M>n$, $m>0$. Note that the Student- and Cauchy-type dfg are special cases of $g_{P T 7}$ and that

$$
\begin{aligned}
I\left(g_{P E}\right) & =p^{n / p-1} \Gamma\left(\frac{n}{p}\right), \\
I\left(g_{K}\right) & =\frac{\Gamma((n+M-1) / \gamma)}{\gamma \beta^{(n+M-1) / \gamma},} \\
I\left(g_{P T 7}\right) & =m^{n} B(M-n, n),
\end{aligned}
$$

where $B$ denotes the beta function. It follows, with $B_{\sigma a, p}=$ $\sigma B_{a, p}$ and

$$
\begin{aligned}
P( & \left.Y-v \in t^{1 / p} B_{\sigma a, p}\right) \\
& =\frac{1}{I(g)} \int_{0}^{\infty} r^{n-1} g(r) \mathfrak{F}_{a, p}\left(t^{1 / p} B_{\sigma a, p}, r\right) d r \\
& =\frac{1}{I(g)} \int_{0}^{t^{1 / p}} r^{n-1} g(r) d r,
\end{aligned}
$$


that, independently of $a$,

$$
\begin{aligned}
& \frac{d}{d t} P\left(n\left(\frac{\widehat{\sigma}}{\sigma}\right)^{p}<t\right) \\
& \quad=\frac{d}{d t} P\left(\left|\frac{Y_{1}-v_{1}}{a_{1}}\right|^{p}+\cdots+\left|\frac{Y_{n}-v_{n}}{a_{n}}\right|^{p}<t\right) \\
& =\frac{t^{n / p-1} g\left(t^{1 / p}\right)}{p I(g)}=f_{n, g, p}^{\chi}(t) .
\end{aligned}
$$

Note that our earlier representation of this density in [13] differs from the present one because of the (slightly) different notation for the dfg $g$.

Example 7. Let $X_{i} \sim f_{p}\left(\cdot ; 0, \sigma_{1}\right), i=1, \ldots, n_{1}$, and $Y_{i} \sim$ $f_{p}\left(\cdot ; 0, \sigma_{2}\right), i=1, \ldots, n_{2}$, where $p>0$ is known and $\sigma_{1}, \sigma_{2}>0$ are unknown. These $\mathrm{rv}$ are assumed to be completely independent. We define $X=\left(X_{1}, \ldots, X_{n_{1}}\right)^{T}$ and $Y=\left(Y_{1}, \ldots, Y_{n_{2}}\right)^{T}$. It follows from the well known theory of exponential families that the statistic $T=\left(|X|_{p}^{p},|Y|_{p}^{p}\right)$ is sufficient for $\left(\sigma_{1}, \sigma_{2}\right)$. The $\operatorname{rv} Z=\left(X^{T} Y^{T}\right)^{T}$ has the density

$$
\begin{array}{r}
f_{Z}\left(\left(x^{T}, y^{T}\right)^{T}\right)=\frac{C_{p}^{n_{1}+n_{2}}}{\sigma_{1}^{n_{1}} \sigma_{2}^{n_{2}}} g_{P E}\left(\left|\left(x^{T}, y^{T}\right)^{T}\right|_{p, \sigma_{1}, \sigma_{2}}\right) \\
x \in \mathbb{R}^{n_{1}}, y \in \mathbb{R}^{n_{2}}
\end{array}
$$

with

$$
\left|\left(x^{T}, y^{T}\right)^{T}\right|_{p, \sigma_{1}, \sigma_{2}}=\left(\frac{|x|_{p}^{p}}{\sigma_{1}^{p}}+\frac{|y|_{p}^{p}}{\sigma_{2}^{p}}\right)^{1 / p}
$$

Thus, for every measurable function $h: \mathbb{R}_{+}^{2} \rightarrow \mathbb{R}$,

$$
P(h(T)<t)=\Phi_{g_{P E}, B_{p, \sigma_{1}, \sigma_{2}}}(B(t)),
$$

$B(t)=\left\{\left(x^{T}, y^{T}\right)^{T}: h\left(|x|_{p}^{p},|y|_{p}^{p}\right)<t\right\}, B_{p, \sigma_{1}, \sigma_{2}}=\left\{\left(x^{T}, y^{T}\right)^{T} \in\right.$ $\left.\mathbb{R}^{n_{1}+n_{2}}:\left|\left(x^{T}, y^{T}\right)^{T}\right|_{p, \sigma_{1}, \sigma_{2}} \leq 1\right\}$.

Applications of formula (46) allow the derivation of geometric measure representations of exact distributions of statistics such as $\alpha|X|_{p}^{p}+\beta|Y|_{p}^{p},|X|_{p}^{p} /|Y|_{p}^{p}$ and $|X|_{p}^{p} /\left(|X|_{p}^{p}+\right.$ $\left.|Y|_{p}^{p}\right)$, under the nonstandard model assumptions made here.

A nonconstant ipf of the set $B(t)$ has been dealt with for different functions $h$ and under different parameter assumptions in earlier papers of the author and several coauthors; see [6].

Example 8. For data in [14] reporting the profits at the box office and the number of sold home videos, in [15] the authors study fitting a linear regression model with random errors distributed according to an exponential power distribution. When analyzing the residuals they present Q-Q-plots for both the exponential power distribution with the estimated parameter $p=2,386877$ and the normal distribution $(p=2)$. The observer's subjective impression after a visual inspection of these plots may be that one cannot really be sure in preferring one of the two error models, this way.

This example, which was presented by the authors only for the purpose of showing the use of the functions implemented by them to fit a linear regression model if errors are possibly exponentially power distributed, gives rise to throwing up in a similar two-dimensional situation the following standard question of statistical practice: how large should a sample size be to make a practical decision based upon a visual inspection "relatively safe"? In particular, how large should a sample size be for the observer being able to visually choose between the two two-dimensional $p$-generalized normal distributions with parameters $p=2$ and $p=2,388677$ ?

This question, clearly, is not formulated in a strong mathematical way and will not be answered in such way, here. Instead, we present Figures 6-8 showing that one can hardly distinguish this way between the parameters $p=2$ and $p=$ 2,388677 of the two-dimensional $p$-generalized normal distribution even if sample sizes are large. As a consequence, one may ask, for example, for a mathematical method yielding a sure decision about the first decimal place of parameter $p$, say. For a certain general 20-percent sensitivity and $g$-robustness principle, established when dealing with another particular problem, we refer to Application 2 and Section 3 in [16].

\section{Example 9.}

(a) Simulation in Dimension One. There are several possibilities for simulating the $p$-generalized normal distribution. The $p$-generalized polar method and the $p$-generalized rejecting polar method are established in [17] and compared with several methods known from the literature. Moreover, the resulting recommendation for using which of the methods in which situation is realized in the R-module "pgnorm"; see [18].

(b) Simulation in Dimension Two. If dimension is $n=2$ and $K=B_{p}$ for some $p>0$ then $S=\left\{\left(x_{1}, x_{2}\right)^{T} \in \mathbb{R}^{2}\right.$ : $\left.\left|x_{1}\right|^{p}+\left|x_{2}\right|^{p}=1\right\}$. A random vector $\left(U_{p, 1}, U_{p, 2}\right)^{T}$ following the star-generalized uniform distribution $\omega_{S}$ on $\mathscr{B}_{S}$ allows the stochastic representation

$$
\left(U_{p, 1}, U_{p, 2}\right)^{T} \stackrel{d}{=}\left(\cos _{p}(\Phi), \sin _{p}(\Phi)\right)^{T},
$$

where the generalized trigonometric functions

$$
\begin{aligned}
\cos _{p}(\varphi) & =\frac{\cos \varphi}{N_{p}(\varphi)}, \\
\sin _{p}(\varphi) & =\frac{\sin \varphi}{N_{p}(\varphi)} \\
& \text { with } N_{p}(\varphi)=\left(|\sin \varphi|^{p}+|\cos \varphi|^{p}\right)^{1 / p}
\end{aligned}
$$

are introduced in [12] and applied to the class of $l_{n, p^{-}}$ symmetric distributions in [13], and the polar angle $\Phi$ has the pdf

$$
f_{\Phi}(\varphi)=\frac{p \Gamma(2 / p)}{4\left(\Gamma(1 / p) N_{p}(\varphi)\right)^{2}} I_{(0,2 \pi)}(\varphi) .
$$




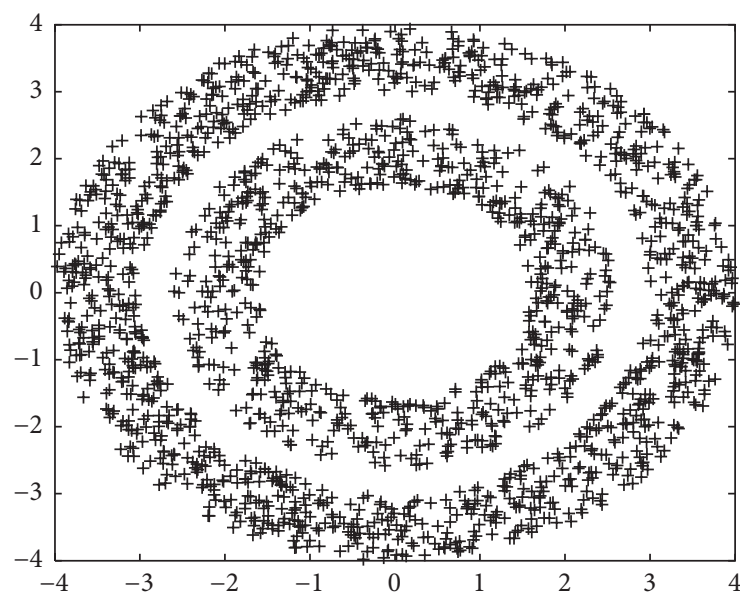

(a) $f_{R}(r)=f(r ; 1 / 3 ; 1.6,2.6 ; 3,4)$



(b) $f_{R}(r)=f(r ; 2 / 3 ; 1.9,2.9 ; 3,4)$

Figure 9: Two-layer $l_{2, p}$-symmetric distributions sampled in $k=2000$ independent trials with $p=2,388677$.

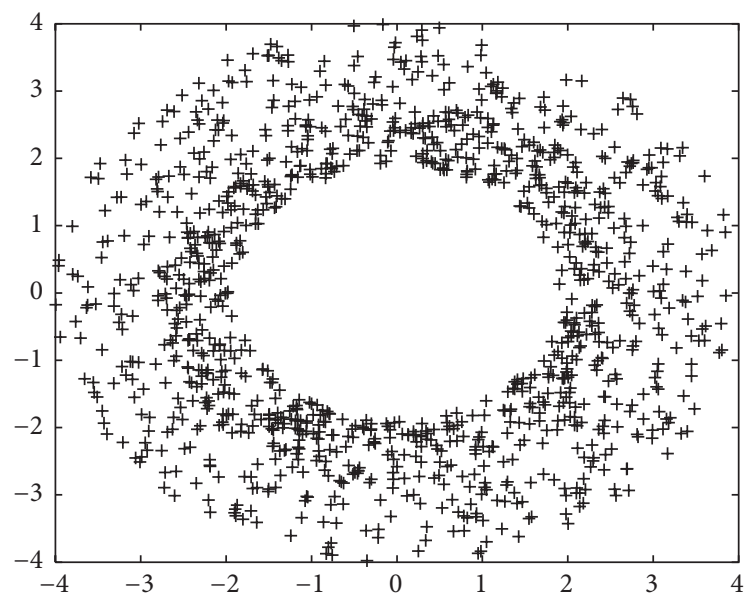

(a) $k=1200$

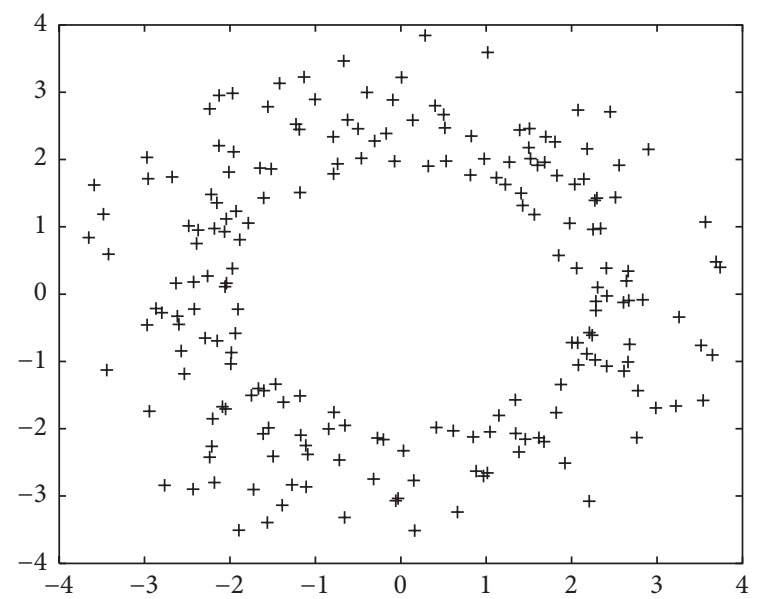

(b) $k=300$

FIGURE 10: Independent realizations of two-layer $l_{2, p}$-symmetrically distributed random points where $p$ and $f_{R}$ are as in Figure $9(\mathrm{~b})$.

For a graphical representation of this function, we refer to [17].

Let $Z$ be uniformly distributed on the interval $(0,1)$, $z_{1}, \ldots, z_{k}$ being realizations of it in $k$ independent trials, and put $F_{\Phi}(\varphi)=\int_{0}^{\varphi} f_{\Phi}(\psi) d \psi, 0<\varphi<2 \pi$. Numerically solving the equations

$$
\begin{aligned}
F_{\Phi}\left(\varphi_{i}\right)=z_{i} \text { with starting value } \varphi_{i, 0}=2 \pi z_{i}, & \\
& i=1, \ldots, k,
\end{aligned}
$$

yields the realizations $\left(u_{p, 1}^{(1)}, u_{p, 2}^{(1)}\right), \ldots,\left(u_{p, 1}^{(k)}, u_{p, 2}^{(k)}\right) \quad$ of $\left(U_{p, 1}, U_{p, 2}\right)^{T}$ from $k$ independent trials.

Moreover, let the random variable $R$ independently of $\left(U_{p, 1}, U_{p, 2}\right)^{T}$ follow the density $f_{R}(r)=f(r ; 1 / 3 ; 1.6,2.6 ; 3,4)$ where, with suitably chosen $a<b<c<d$,

$$
\begin{aligned}
& f(r ; p ; a, b ; c, d)=p I_{(a, b)}(r)+(1-p) I_{(c, d)}(r), \\
& 0<p<1 .
\end{aligned}
$$

If $r_{1}, \ldots, r_{k}$ are realizations of $R$ in independent trials then $y_{i}=r_{i}\left(u_{p, 1}^{(i)}, u_{p, 2}^{(i)}\right), i=1, \ldots, k$, are realizations of a so-called two-layer $l_{2, p}$-symmetrically distributed random vector $Y$ which are represented in Figure 9(a). Similarly, Figure 9(b) is drawn with $f_{R}(r)=f(r ; 2 / 3 ; 1.9,2.9 ; 3,4)$. Still using the latter function but having smaller sample sizes, Figure 10 is generated. It turns out that it becomes impossible to further visually distinguish between the two layers for sample sizes becoming too small.

We remark additionally that Figures 9 and 10 do not represent elliptically contoured distributions as one might argue at first glance. This again supports the above discussion on introducing 20-percent $g$-robust decisions.

Finally, we notice that Figures 1-8 are generated with the level density function of the two-dimensional $p$-generalized normal density:

$$
f_{R}(r)=\frac{1}{p^{2 / p-1} \Gamma(2 / p)} r e^{-r^{p} / p}, \quad r>0 .
$$




\section{Proofs}

The general method of proof in this paper can be divided into two main parts. Using the properties of the support function of a convex body, $h_{K}$, in the first part it will be shown that the absolute value of the Jacobian of a certain transformation may be interpreted in terms of the normal vector $N$ to the boundary of $K$. This allows according to Lemma 1 in [6] representing the surface measure $\mathfrak{D}_{S}$ as an integral of $h_{K}(N)$. The second part of the method of proof deals with a relation between the functional $h_{K}(N)$ and the Minkowski functional of a suitably defined set $K^{*}$, or $K^{o}$. While, in the convex case, $K^{*}$ is extensively studied, $K^{o}$ yet has to be found in the most general case when $K$ is radially concave with respect to a fan in $\mathbb{R}^{n}$.

Proof of Theorem 2. The support function of the convex body $K$ is defined as

$$
h_{K}(u)=\sup \left\{u^{T} y: y \in K\right\}, \quad u \in \mathbb{R}^{n} .
$$

Recall that if $u \in S^{(n-1)}$ then $h_{K}(u)$ describes the distance from the origin to the hyperplane with outer normal vector $u$ and supporting $K$. For compactness and continuity reasons, the supremum is always attained:

$$
\forall u \in S^{(n-1)} \exists x_{S}(u) \in S: h_{K}(u)=u^{T} x_{S}(u) .
$$

The set of all such points $x_{S}$ is called the supporting set of $K$ at $u$. If the norm is smooth then $x_{S}(u) \in S \cap T(u)$ where $T(u)$ is the tangent hyperplane to $S$ at the point $x_{S}(u)$ with $T(u)$ being orthogonal to $u$. If $K$ is strongly convex then the supporting set of $K$ at $u$ consists of just one point, thus $x_{S}(u)$ being then always uniquely defined. Note that it may happen that a point $\xi \in S$ satisfies $\xi=x_{S}(u)$ for more than one point $u \in S^{(n-1)}$. To see this, assume that $S$ contains corner points and let $\xi$ be such a corner point of $S$. As a consequence, even the union of all supporting sets of a convex body may be finite.

According to Lemma 1 in [6],

$$
\mathfrak{D}_{S}(A)=\int_{G(A)}\left|\left(\vartheta^{T}, \eta(\vartheta)\right) N(\vartheta)\right| d \vartheta,
$$

where the function $\vartheta \mapsto \eta(\vartheta)$ is chosen such that $d_{K}\left(\left(\vartheta^{T}\right.\right.$, $\left.\eta(\vartheta))^{T}\right)=1$ describes the boundary $S$ of $K$. Note that here $x=\left(\vartheta^{T}, \eta(\vartheta)\right)^{T} \in S$ and a.e. $N(\vartheta)=(\nabla \eta(\vartheta),-1)^{T}$ is the outer normal vector of $S$ at $\left(\vartheta^{T}, \eta(\vartheta)\right)^{T}$, and thus $\left(\vartheta^{T}, \eta(\vartheta)\right) N(\vartheta)>0$. It follows from (54) that

$$
\begin{aligned}
h_{K}(n(\vartheta)) & =n(\vartheta)^{T}\left(\vartheta^{T}, \eta(\vartheta)\right)^{T}, \\
n(\vartheta) & =\frac{N(\vartheta)}{|N(\vartheta)|_{E}},
\end{aligned}
$$

where $|\cdot|_{E}$ means the Euclidean norm. By the homogeneity property of $h_{K}$,

$$
\mathfrak{D}_{S}(A)=\int_{G(A)} h_{K}(N(\vartheta)) d \vartheta .
$$

The theorem follows by the well known fact that $h_{K}=d_{K^{*}}$.
Proof of Theorem 3. We consider $\left(\vartheta^{T}, \eta(\vartheta)\right)^{T} \in S \cap C_{i}$ and denote the (a.e. defined) inner normal vector to the boundary $S$ of the antinorm ball $K$ at $\left(\vartheta^{T}, \eta(\vartheta)\right)^{T}$ by $N(\vartheta)$. Further we put $n(\vartheta)=N(\vartheta) /|N(\vartheta)|_{2}$ and $u=-n(\vartheta)$. Then $u \in S^{(n-1)} \cap C_{i}$ and because $K \in \mathrm{AN} 1$,

$$
\begin{aligned}
\left|\left(\vartheta^{T}, \eta(\vartheta)\right)^{T} n(\vartheta)\right| & =\left|x_{S, i}^{T}(n(\vartheta)) n(\vartheta)\right| \\
& =\inf \left\{n^{T}(\vartheta) y: y \in S \cap C_{i}\right\} .
\end{aligned}
$$

Thus, $\left|\left(\vartheta^{T}, \eta(\vartheta)\right)^{T} n(\vartheta)\right|=h_{K}^{\mathfrak{\mho}}(n(\vartheta))$, and

$$
\mathfrak{D}_{S}(A)=\int_{G(A)} h_{K}^{\mathfrak{F}}(N(\vartheta)) d \vartheta .
$$

The proof will be finished by the following lemma.

Lemma 10. The antisupport function of $K$ with respect to $\mathfrak{F}$ is equal to the distance function of $K^{o}, h_{K}^{\mathfrak{F}}=d_{K^{o}}$.

Proof. The radial function of the radially concave star-shaped set $K^{o}$ is, on the one hand, by definition

$$
\varrho_{K^{o}}(u)=\sup \left\{\lambda \geq 0: \lambda u \in K^{o}\right\}=\frac{1}{h_{K}^{\mathfrak{F}}(u)}, \frac{}{u \in S^{(n-1)},}
$$

and allows, on the other hand, the representation

$$
\begin{aligned}
\varrho_{K^{o}}(u) & =\sup \left\{\lambda \geq 0: u \in \frac{1}{\lambda} K^{o}\right\} \\
& =\frac{1}{\inf \left\{\mu \geq 0: u \in \mu K^{o}\right\}}=\frac{1}{d_{K^{o}}(u)} .
\end{aligned}
$$

Thus $h_{K}^{\mathfrak{F}}=d_{K^{o}}$.

\section{Discussion}

To make both the similarity and the difference between Theorems 2 and 3 more visible, let us remark that $K^{*}$ allows a representation looking similar to that of $K^{o}$ :

$$
\begin{aligned}
K^{*} & =\left\{y \in \mathbb{R}^{n}: y^{T} x \leq 1, \forall x \in K\right\} \\
& =\left\{\lambda(u) u: 0 \leq \lambda(u) h_{K}(u) \leq 1, u \in S^{(n-1)}\right\} .
\end{aligned}
$$

For dealing with a combined example where both Theorems 2 and 3 apply, we recall that the function $x \mapsto|x|_{a, p}$ is a norm if $p \geq 1$ and, according to [11], an antinorm if $0<p \leq 1$. Thus, $K=B_{a, p}$ is convex if $p \geq 1$ and radially concave with respect to the standard fan $\mathfrak{F}$ if $0<p \leq 1$. Let $q$ be defined by the equation $1 / p+1 / q=1$, and $1 / a=\left(1 / a_{1}, \ldots, 1 / a_{n}\right)^{T}$. Note that if $p>1$ then $K^{*}=B_{1 / a, q}$, and if $0<p<1$ then $q<0$ and

$$
K^{o}=\left\{x \in \mathbb{R}^{n}: \sum_{i=1}^{n} \frac{1}{\left|a_{i} x_{i}\right|^{|q|}} \geq 1\right\}
$$


is a semiantinorm ball. For an illustration of such sets, see [11]. Note that $a$ and $p$ are independently dealt with when constructing the sets $K^{*}$ and $K^{o}$.

Remark 11. In the applications of Theorem 2, the surface content of $[(1 / r)(B-v)] \cap S$ (which may be considered as an indivisible of the set $B-\nu$ ) is always measured with respect to the metric $d_{K^{*}}$. Successful applications of this generalized method of indivisibles are surveyed in [6]. The representation of Theorem 2 generalizes those presented in the latter and several earlier papers.

Remark 12. The set $K^{o}$ in Section 3.2 is radially concave; thus $d_{K^{o}}$ is a semiantinorm.

Proof. We show that if $x_{1}$ and $x_{2}$ are from $\bar{K}^{o} \cap C$ for some $C \in \mathfrak{F}$ then $\lambda x_{1}+(1-\lambda) x_{2} \in \bar{K}^{o} \cap C$ for $0<\lambda<1$, where $\bar{K}^{o}$ means the complement of the set $K^{o}$. Let $x_{1}=\lambda_{1} u_{1}, x_{2}=$ $\lambda_{2} u_{2}$ with $u_{i} \in S^{(n-1)}, \lambda_{i} \geq 1 / h_{K}^{\mathfrak{F}}\left(u_{i}\right), i=1,2$. Then

$$
\begin{aligned}
h_{K}^{\mathfrak{F}} & \left(\lambda x_{1}+(1-\lambda) x_{2}\right) \\
& =h_{K}^{\mathfrak{F}}\left(\lambda \lambda_{1} u_{1}\right)+h_{K}^{\mathfrak{F}}\left((1-\lambda) \lambda_{2} u_{2}\right) \\
& \geq \frac{\lambda \lambda_{1}}{\lambda_{1}}+\frac{(1-\lambda) \lambda_{2}}{\lambda_{2}}=1 .
\end{aligned}
$$

\section{Conflict of Interests}

The author declares that there is no conflict of interests regarding the publication of this paper.

\section{Acknowledgment}

The author is grateful to Klaus Müller for his help in drawing the figures during the necessarily fast revision process.

\section{References}

[1] K.-T. Fang and Y. Zhang, Generalized Multivariate Analysis, Springer, Berlin, Germany, 1990.

[2] T. W. Anderson and K.-T. Fang, "Theory and applications of elliptically contoured and related distributions," Technical Report 24, Department of Statistics, Stanford University, Stanford, Calif, USA, 1990.

[3] M. Bilodeau and D. Brenner, Theory of Multivariate Statistics, Springer, New York, NY, USA, 1999.

[4] E. Liebscher, "A semiparametric density estimator based on elliptical distributions," Journal of Multivariate Analysis, vol. 92, no. 1, pp. 205-225, 2005.

[5] N. Balakrishnan and C.-D. Lai, Continuous Bivariate Distributions, Springer, 2009.

[6] W.-D. Richter, "Geometric disintegration and star-shaped distributions," Journal of Statistical Distributions and Applications, vol. 1, article 20, 2014.
[7] M. Rudemo and H. Stryhn, "Boundary estimation for starshaped objects," in Change-Point Problems, E. Carlstein, H.G. Müller, and D. Siegmund, Eds., pp. 276-283, Institute of Mathematical Statistics, Hayward, Calif, USA, 1994.

[8] A. B. Tsybakov, "On nonparametric estimation of density level sets," The Annals of Statistics, vol. 25, no. 3, pp. 948-969, 1997.

[9] G. Walther, "Granulometric smoothing," The Annals of Statistics, vol. 25, no. 6, pp. 2273-2299, 1997.

[10] A. Cuevas, M. Febrero, and R. Fraiman, "Estimating the number of clusters," Canadian Journal of Statistics, vol. 28, no. 2, pp. 367$382,2000$.

[11] M. Moszyńska and W.-D. Richter, "Reverse triangle inequality. Antinorms and semi-antinorms," Studia Scientiarum Mathematicarum Hungarica, vol. 49, no. 1, pp. 120-138, 2012.

[12] W.-D. Richter, "Generalized spherical and simplicial coordinates," Journal of Mathematical Analysis and Applications, vol. 336, no. 2, pp. 1187-1202, 2007.

[13] W.-D. Richter, “Continuous $l_{n, p}$-symmetric distributions," Lithuanian Mathematical Journal, vol. 49, no. 1, pp. 93-108, 2009.

[14] D. M. Levine, T. C. Krehbiel, and M. L. Berenson, Business Statistics: A First Course, Prentice-Hall, 2000.

[15] A. M. Mineo and M. Ruggieri, "A software tool for the exponential power distribution: the normal package," Journal of Statistical Software, vol. 12, no. 4, pp. 1-24, 2005.

[16] C. Ittrich, D. Krause, and W.-D. Richter, "Probabilities and large quantiles of noncentral generalized chi-square distributions," Statistics, vol. 34, no. 1, pp. 53-101, 2000.

[17] S. Kalke and W.-D. Richter, "Simulation of the p-generalized Gaussian distribution," Journal of Statistical Computation and Simulation, vol. 83, no. 4, pp. 639-665, 2013.

[18] S. Kalke, "pgnorm," The R Project for Statistical Computing, https://www.r-project.org. 


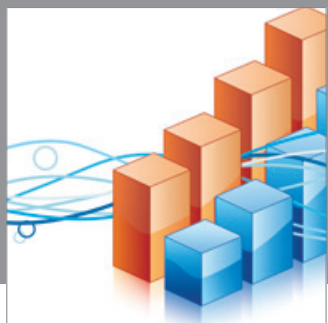

Advances in

Operations Research

mansans

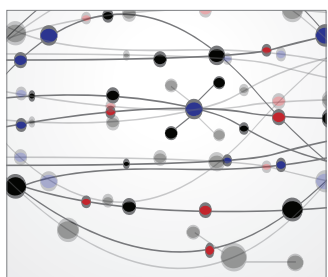

The Scientific World Journal
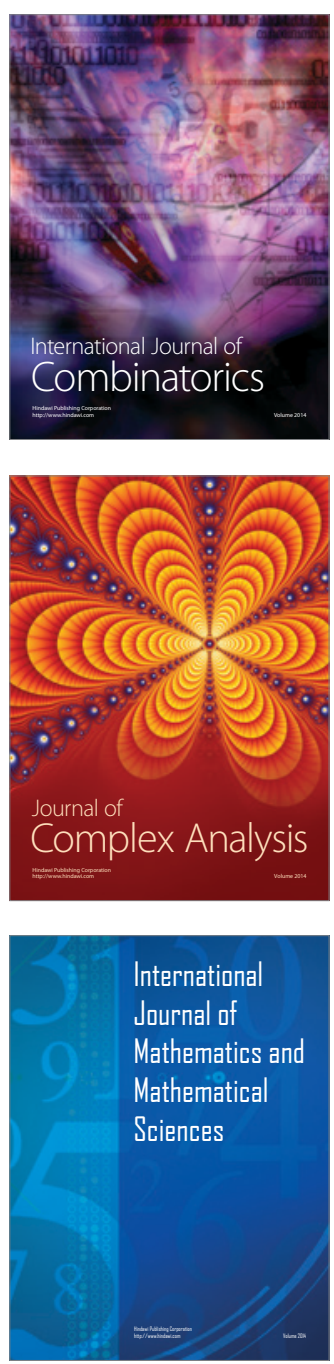
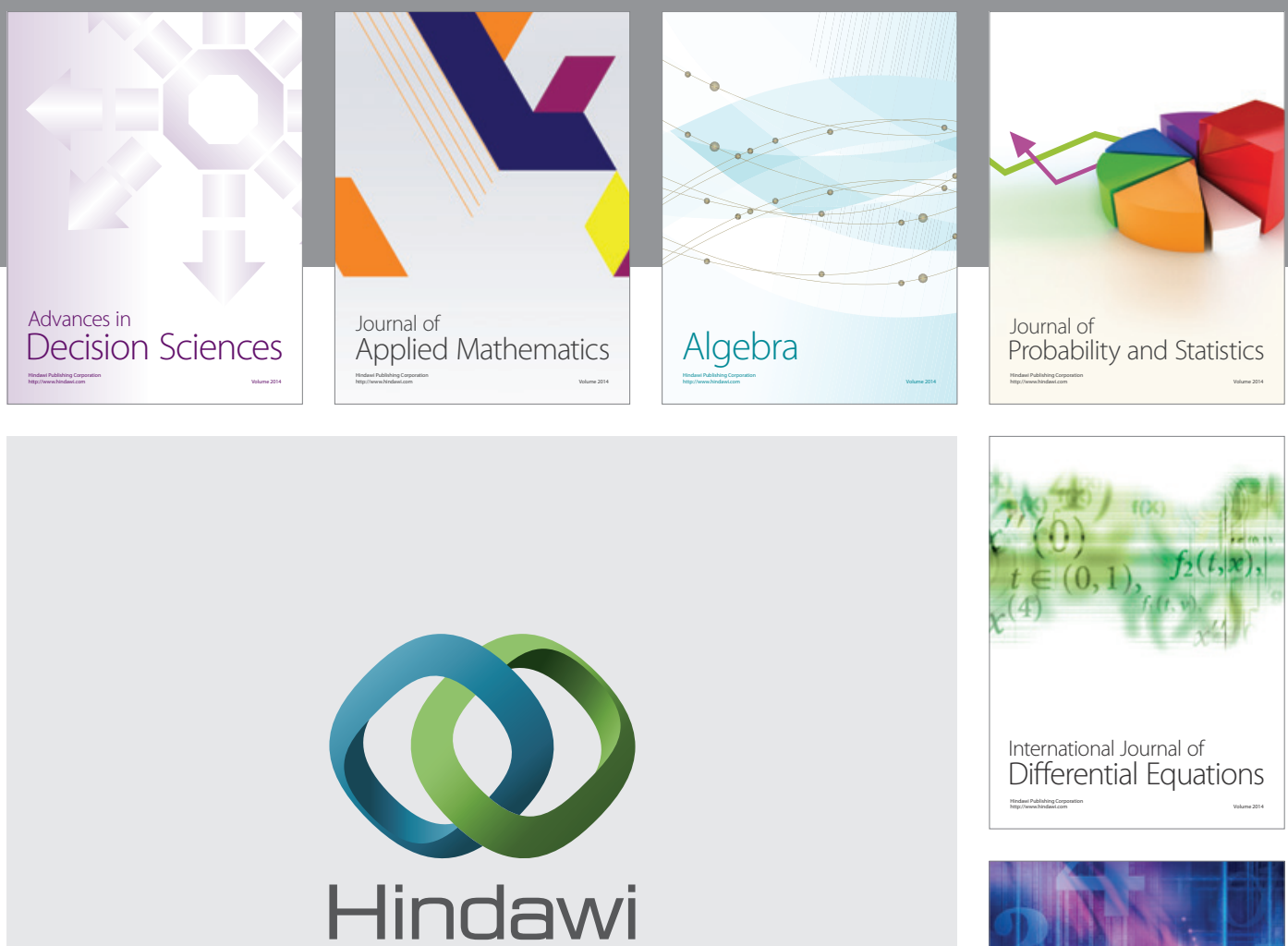

Submit your manuscripts at http://www.hindawi.com
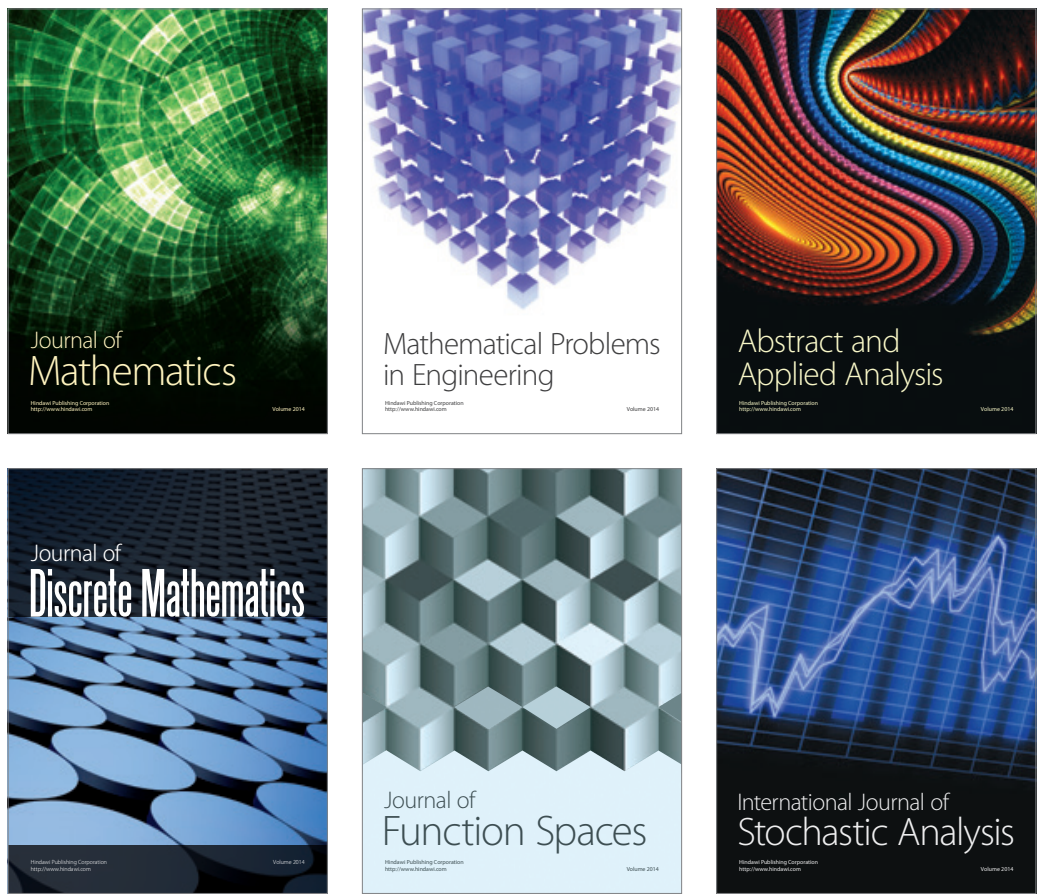

Journal of

Function Spaces

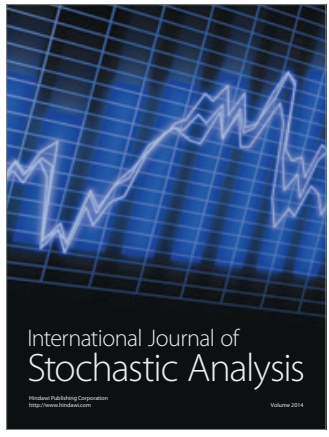

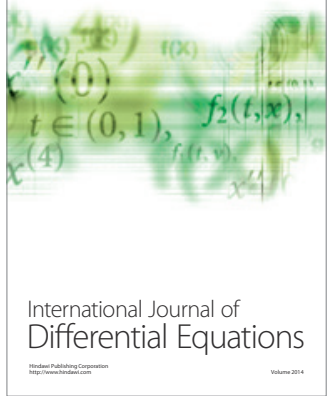
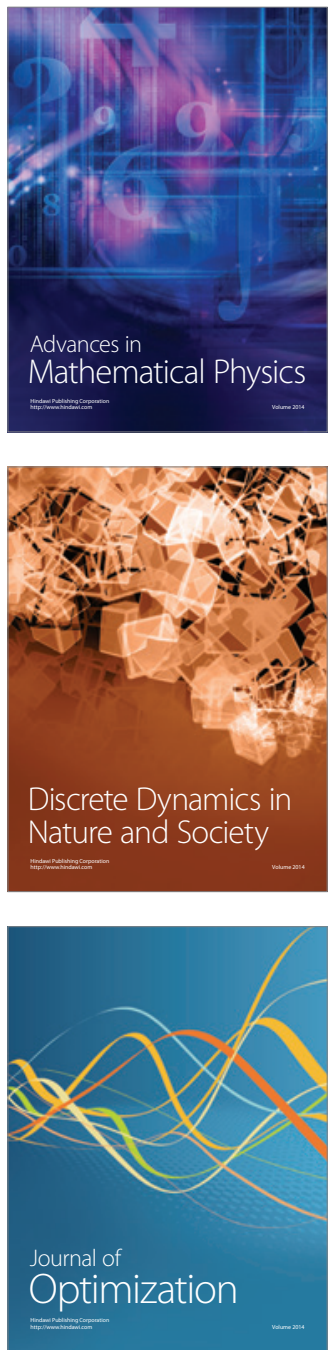